Cita bibliográfica: Morales Hernández, A. y Fernández Hernández, C. (2019). Acceso a la autonomía económica de las mujeres a través del turismo rural en la isla de La Palma Investigaciones Turísticas (18), pp. 22-41. http:// dx.doi.org/10.14198/INTURI2019.18.02

\title{
Acceso a la autonomía económica de las mujeres a través del turismo rural en la isla de La Palma
}

\section{Access of women to economic autonomy through rural tourism on the island of La Palma}

Ana María Morales Hernández (iD, Universidad de La Laguna, España ana.morales.hdez@gmail.com

Carlos Fernández Hernández iD, Universidad de La Laguna, España cferher@ull.edu.es

\section{RESUMEN}

La división de género del mercado de trabajo en los espacios rurales repercute en el turismo rural. Las mujeres participan en menor medida que los hombres del mercado laboral y, cuando lo hacen, ocupan en mayor proporción puestos de trabajo temporal o a tiempo parcial, orientados al sector servicios. Estas características están relacionadas con la discontinuidad y flexibilidad de la demanda turística, así como con el tipo de labores que requiere la oferta de alojamiento de turismo rural. A través de un estudio cuantitativo de las personas propietarias de casas rurales de la isla de La Palma (Canarias, España), se observa que las mujeres reciben un significativo mayor porcentaje de ingresos a partir del turismo rural que los hombres. Sin embargo, estos ingresos no son suficientes como para garantizar una renta completa que permita su autonomía económica a través del turismo rural. Los resultados de este trabajo sostienen como certeza la segregación de género en el mercado laboral rural. Las evidencias obtenidas afianzan el hecho de una significativa mayor presencia de mujeres que de hombres ejerciendo el turismo rural como principal ocupación. Si bien el turismo rural no altera sustancialmente el modelo de desempeño imperante, nutriéndose de unas experiencias adquiridas por las mujeres en el ámbito doméstico y sin llegar a reportar una renta completa, supone para ellas cierto avance en el grado de su autonomía. Estos aspectos deben ser base para una acción pública que fomente el potencial de desarrollo del turismo rural desde una mayor equidad de género.

Palabras clave: género, mercado laboral rural, turismo rural, autonomía económica. 


\section{ABSTRACT}

The gender division in the labour market in rural areas has repercussions on rural tourism. Women participate to a lesser extent than men in the labour market and, when they do, they occupy a greater proportion of temporary or part-time jobs, oriented to the service sector. These characteristics are related to the discontinuity and flexibility of tourist demand, as well as the type of tasks required by the accommodation offer of rural tourism. Through a quantitative study of the owners of rural houses on La Palma (Canary Islands, Spain), it can be observed that women receive a significantly higher percentage of income from rural tourism than men. However, this revenue is not enough to ensure a full income that allows their economic autonomy through rural tourism. The results of this study support gender segregation in the rural labour market. The evidence obtained strengthens the fact that there is a significantly higher presence of women than men exercising rural tourism as their main occupation. Although rural tourism does not substantially alter the prevailing performance model, drawing from experiences acquired by women in the domestic sphere and without reporting a full income, this represents a certain level of progress in their degree of autonomy. These aspects should constitute the basis for public action that promotes the potential for rural tourism development with a higher degree of gender equity.

Keywords: gender, rural labour market, rural tourism, economic autonomy.

\section{INTRODUCCIÓN}

La autonomía económica de las mujeres en los espacios rurales está estrechamente relacionada con el tipo de participación que realizan en el mercado laboral remunerado de los entornos rurales, lo cual determina sus posibilidades de independencia y sus condiciones de vida. La tradicional división del trabajo a partir de la asignación de distintos roles y tareas en función del género de los miembros del grupo familiar ha dificultado su acceso a recursos y oportunidades, de manera más acusada en el medio rural. Tradicionalmente, los hombres han asumido el trabajo productivo, con la generación de ingresos económicos y el reconocimiento público de sus labores, mientras que las mujeres han quedado relegadas al desarrollo de trabajos reproductivos y empleos temporales y parciales, sin la suficiente estabilidad económica y visibilidad social de las tareas que realizan.

De esta forma, se encuentra una brecha de género en el mercado laboral de las zonas rurales (Alonso \& Trillo, 2014). En esta línea, la propia ruralidad ha obstaculizado el acceso de las mujeres a empleos remunerados, no sólo por la mayor escasez de servicios básicos de atención a la infancia y personas dependientes que dificulta la conciliación laboral y familiar, sino también por las expectativas culturales y las normas sociales con respecto al papel que tradicionalmente se les ha asignado como deber a cumplir en el cuidado de sus familias. Así, se han restringido sus oportunidades de acceso al mercado de trabajo remunerado, a la par que sus aspiraciones profesionales han tenido un papel secundario (Little \& Panelli, 2003).

No obstante, las contribuciones productivas de las mujeres han sido fundamentales para el desarrollo socioeconómico de los entornos rurales. Principalmente, las mujeres rurales se dedicaban a las tareas de explotación agraria en calidad de "ayuda familiar", aunque sin 
la correspondiente remuneración y cotización a la Seguridad Social (Vera Toscano, 2009), así como a la elaboración de productos artesanales, en muchos casos desde la economía sumergida, el trabajo en negocios familiares, o como asalariadas del sector agrícola y ganadero, y de la industria alimentaria de manera estacional (Langreo Navarro y Benito García, 2005).

A partir de los años noventa, se observa un salto cualitativo en la incorporación de las mujeres al trabajo productivo remunerado, especialmente, en el grupo comprendido entre 25 y 45 años de edad (Alario, Baraja y Pascual, 2008). La asalarización del trabajo femenino ha sido la principal característica de la participación de las mujeres en el mercado laboral (Cànoves Valiente y Blanco Romero, 2008). Los decrecientes rendimientos económicos de la agricultura tradicional, así como los cambios en los modos de vida y el incremento del consumo fueron factores que hicieron necesaria la incorporación de nuevos ingresos, a través del salario femenino, a la unidad familiar (García Sanz, 2009; Viruela Martínez y Domingo Pérez, 2000). Asimismo, las mujeres encontraron mayores alternativas de empleo como resultado del mejoramiento de su nivel formativo y del aumento de las oportunidades de trabajo a través de la terciarización de la economía, ajustando sus necesidades y aspiraciones a las nuevas oportunidades ofrecidas.

El sector servicios ha sido fundamental para la fijación de población en el medio rural, como alternativa laboral ante los procesos de reestructuración económica (Gómez García y Rico González, 2005). Así, mientras la agricultura ha tendido a la masculinización, los servicios han manifestado una propensión a su feminización. Se trata de una combinación del trabajo doméstico con la creación de una actividad que genera ingresos (Rijkers \& Costa, 2012) o una "asalarización de funciones" que las mujeres han ejercido tradicionalmente como trabajo reproductivo en el espacio doméstico (Viruela Martínez et al., 2000), manteniendo la división de género del trabajo a través de una segregación horizontal de la ocupación que orienta a las mujeres, principalmente, a labores de servicio y cuidados, hostelería, comercio, educación y sanidad (Alario et al., 2008).

A su vez, la inserción laboral de las mujeres rurales continúa marcada por las exigencias de sus responsabilidades reproductivas y las posibilidades de conciliación familiar y laboral. La maternidad es un factor que influye enormemente en el abandono de las mujeres del mercado de trabajo remunerado (Gómez García et al., 2005). De este modo, la participación laboral de las mujeres se define en base a su identidad de género, en tanto su vida laboral se encuentra interrumpida por las responsabilidades reproductivas que condicionan su capacidad para asumir el trabajo remunerado (Cànoves Valiente et al., 2008). Asimismo, la percepción de la contribución efectiva de los ingresos económicos de las mujeres a la economía de sus familias puede tener repercusiones en las relaciones de género del ámbito familiar, tanto por el surgimiento del conflicto de roles como por la necesidad de llegar a nuevos acuerdos para el mantenimiento de la organización familiar (Forstner, 2013).

Como resultado de la necesidad de compatibilizar el trabajo reproductivo, se observa una notable ocupación de las mujeres en trabajos temporales y a tiempo parcial en los entornos rurales (Censo de Población y Vivienda, 2011), así como su menor presencia en puestos de alta responsabilidad, lo cual aumenta las condiciones de precariedad laboral y peor remuneración y reconocimiento de su trabajo productivo (Millán Vázquez, Velasco y Ramírez, 2017, Moreno Mínguez, 2003; Vera Toscano, 2009). Asimismo, las mujeres están expuestas a situaciones de 
discriminación laboral que resultan en una brecha salarial, al recibir menor salario que los hombres por el desempeño de un mismo empleo, debido a la infravaloración del trabajo que realizan (Millán Vázquez et al., 2017). Las realidades de subempleo, inestabilidad y precariedad laboral que experimentan las mujeres rurales en base a su condición de género, se agudizan al combinarse con otros factores como la edad, el origen cultural, la cualificación y la cultura de género de las parejas y la sociedad rural (Suárez Ortega, 2016). Además, la falta de servicios básicos, agravada en períodos de crisis, repercute en el retorno a los tradicionales roles de género, perjudicando su posición en el mercado de trabajo remunerado.

En cuanto al emprendimiento femenino posibilitado por la diversificación económica del medio rural, se observa que el autoempleo se ha convertido en una estrategia para afrontar las dificultades de empleo en estos entornos, facilitando a su vez la flexibilidad necesaria para la compatibilización de las responsabilidades familiares (García Sanz, 2009; Pallarès, Tulla y Vera, 2015). Sin embargo, los emprendimientos de las mujeres en el medio rural, a pesar de romper con el modelo de herencia de los negocios familiares por la vía masculina, continúan condicionados por el proyecto familiar, lo cual, pese a suponer una alternativa, también conforma una limitación (Millán Vázquez et al., 2017; Sampedro y Camarero, 2007). Además, los emprendimientos de mujeres tienden a ser de menor tamaño y productividad, y tienen mayor probabilidad de mantenerse en el ámbito familiar, que aquellos impulsados por hombres (Pallarès et al., 2015; Rijkers et al., 2012). Asimismo, se trata, especialmente, de cualificaciones empresariales que las mujeres han aprendido y experimentado en el espacio doméstico, tal como la alimentación, el hospedaje y las artesanías, y cuyas prioridades de emprendimiento van más allá de objetivos económicos, como la autorrealización y la satisfacción personal de contribuir a la economía familiar (Anthopoulou, 2010).

En definitiva, la participación de las mujeres rurales en el mercado de trabajo remunerado puede suponer el acceso a una fuente de ingresos independiente, así como a una serie de derechos y oportunidades personales y profesionales. Partiendo de los específicos condicionamientos que influyen en la situación laboral de las mujeres en los entornos rurales, a través de este estudio se realiza una aproximación al papel desempeñado por el turismo rural en el mejoramiento de la autonomía económica y personal de las mujeres que participan en esta actividad productiva en el medio rural.

\section{ANTECEDENTES TEÓRICOS}

\subsection{Las contribuciones de las mujeres al emprendimiento en turismo rural}

Una de las actividades fundamentales que ha impulsado la reestructuración económica y social de las zonas rurales ha sido el turismo rural. En esta actividad, las mujeres rurales han encontrado un espacio donde diversificar sus economías (Alonso et al., 2014), favoreciendo la mejora de sus ingresos y amortiguando la necesidad de un éxodo rural, a la par que fomentando la dinamización del medio rural.

A través del turismo rural, las mujeres han podido acceder a una posibilidad de empleo, así como a una fuente de ingresos que mejora su nivel de vida y el de sus familias (Caballé Rivera, 2000; García-Ramón, Cànoves, Salamaña, Valdovinos y Villamarino, 1995; Kiper, Ozdemir \& Basaran, 2011). El acceso a ingresos económicos a través del turismo rural 
permite a las mujeres obtener un mayor control sobre las decisiones económicas al interior de sus familias (Wilkinson \& Pratiwi, 1995), participando del prestigio derivado de la contribución financiera en el hogar, lo cual estimula el desarrollo de una identidad propia y una representación positiva de sí mismas (Tajeddini, Ratten \& Denisa, 2017), a la par que se mejora el bienestar de los hogares rurales. Además, la tendencia al trabajo de las mujeres en turismo rural se extiende a otras actividades económicas relacionadas, como son la artesanía y la alimentación tradicional (Khatiwada \& Silva, 2015). Paralelamente, las mujeres emprendedoras en turismo rural ven incrementada su autoestima y confianza personal, y encuentran en la actividad una oportunidad para abrir sus horizontes al establecer contacto con personas de diferentes culturas y modos de pensar (lakovidou, 2002).

La participación de las mujeres en el turismo rural ha sido fundamental, en tanto las tareas que requiere para su desarrollo son semejantes a las labores domésticas que tradicionalmente han llevado a cabo en el hogar (García-Ramón et al., 1995). El cuidado de sus familias y hogares, que normalmente han acostumbrado a realizar, convierte a las mujeres rurales, desde los ojos de sus familias y comunidades, en las "mejor preparadas" para las actividades de servicio al visitante y mantenimiento de la casa rural (Caballé Rivera, 2000; Dávila, Pinuer y Szmulewicz, 2011). La creación de un entorno hogareño, la decoración del lugar y la preparación de las comidas son tareas que se arraigan en las competencias tradicionales de las mujeres, las cuales no han sido aprendidas mediante la educación formal, sino a través de la transmisión cultural (Brandth, Haugen \& Kroken, 2011).

De esta forma, las mujeres han ocupado un papel relevante en el ejercicio de la actividad turística en las zonas rurales, bajo la percepción de que su trabajo en el turismo rural es una extensión del trabajo doméstico que normalmente les ha tocado asumir en sus hogares (García-Ramón et al., 1995). Con esto, se manifiesta una asignación de funcionalidades diferentes para mujeres y hombres en el desarrollo del turismo rural (Rodríguez Muñoz y Acevedo Duarte, 2015). Así, mientras los hombres realizan mayoritariamente actividades de exterior, las mujeres son las principales encargadas de llevar a cabo las labores del interior de las unidades domésticas, tal y como son la limpieza de sus instalaciones y la elaboración de los alimentos.

En efecto, pese a que el turismo rural permite visibilizar las labores cotidianas que las mujeres rurales realizan, otorgándoles una parcela para su autoexpresión, el factor espacial que las confina al ámbito reproductivo no favorece su emancipación (Annes \& Wright, 2015). Las mujeres no alcanzan una igualdad de oportunidades con respecto a los hombres desarrollando su actividad al interior de la unidad familiar, puesto que esto se traduce en una circulación continua entre las esferas doméstica y profesional.

Aunque un conjunto de mujeres promotoras de turismo rural puede representarse con mayor autonomía económica que con anterioridad a su trabajo en la actividad turística, su posición no parece presentar mejoría con respecto a la situación de sus compañeros varones (Feng, 2013). A esto se une la fluctuante estacionalidad turística, lo cual convierte las entradas económicas de las mujeres promotoras de turismo rural en complementos inestables y dependientes de otros ingresos principales de la economía familiar.

No obstante, el acceso de las mujeres al trabajo en el turismo rural les ofrece ventajas específicas que permiten mitigar las desigualdades de género (Khatiwada et al., 2015). Pese a 
que el turismo rural no ha logrado conseguir relaciones de género igualitarias, las mujeres han desafiado las dinámicas de género en el medio rural, al ser las principales artífices del emprendimiento de una actividad propia que va más allá de su tradicional condición de ayuda familiar en las explotaciones agrarias (Wright \& Annes, 2014). A través del desarrollo del turismo rural, las mujeres hacen públicos sus conocimientos y habilidades en la nueva representación de la ruralidad. Igualmente, a pesar de llevar a cabo tareas domésticas de cocina y limpieza, requieren de la colaboración de sus compañeros varones, que de manera transgresora pasan a ocupar la categoría de ayudantes de su actividad (Brandth et al., 2010).

De la misma manera, las mujeres rurales valoran la oportunidad de trabajo que les brinda el turismo rural y la mayor visibilidad de su contribución económica a las familias, a pesar de que la actividad no ha consolidado la profesionalización de su ocupación, debido a los ingresos complementarios que proporciona y las labores domésticas que requiere (GarcíaRamón et al. 1995). Además, las mujeres aprecian la obtención de ingresos económicos sin alejarse del hogar, lo cual les permite combinar las responsabilidades familiares y profesionales a partir de la flexibilidad que proporcionan los pequeños negocios familiares (Tajeddini et al., 2017). Así, pese a la mayor carga de trabajo que experimentan las mujeres que desarrollan la actividad turística en el medio rural, en relación al resto de responsabilidades adscritas a su género, las emprendedoras han encontrado en el turismo rural un medio de acceso a mayores cotas de independencia económica (Wilkinson et al., 1995). De esta forma, se observa cómo el impacto del turismo rural sobre las mujeres promotoras fluye entre aguas tan dispares como el mantenimiento de la división de género del trabajo y el empoderamiento económico de las mismas, de manera que, a pesar de conseguir ciertas cotas de autonomía económica, estas se encuentran insertas en una dinámica de dependencia de los roles de género, bajo el mandato de la estructura patriarcal (Annes et al., 2015).

En lo que respecta a la evaluación de los resultados de las empresas de turismo rural gestionadas por mujeres, se debe tener en cuenta la doble función productiva y reproductiva que realizan cuando se reflexiona sobre su éxito empresarial en la actividad (Diéguez, Gueimonde y Sinde, 2010), puesto que la percepción de las personas empresarias también presenta diferencias en función de la distinta socialización de los géneros. Es importante abordar análisis que no involucren exclusivamente medidas financieras de ingresos y beneficios, sino también parámetros cualitativos que tienen gran valor, especialmente, para las mujeres, por el doble rol que asumen, como son el control del entorno laboral, la compatibilización de las tareas reproductivas y la realización personal (Anthopoulou, 2010).

\section{METOdOLOGÍA}

El principal objetivo de este estudio es analizar la capacidad de autonomía económica que tienen las mujeres promotoras de turismo rural en la isla de La Palma a través del ejercicio de la actividad. A su vez, se plantean como objetivos secundarios establecer un perfil de las personas promotoras de turismo rural en la isla, así como conocer el trabajo requerido para el desempeño de la actividad. Para ello, se recurrirá a comparar la situación de las mujeres propietarias o promotoras de la actividad con respecto a la situación de los hombres dedicados al turismo rural. 
La investigación realizada se contextualiza en la isla de La Palma (Archipiélago de Canarias, España). La elección de este marco de estudio se asienta en diversas razones que se relacionan con las características singulares de este territorio. En La Palma se observa un modelo de desarrollo turístico diseminado, inspirado en el originario turismo rural que emerge a finales de los años ochenta e inicios de los noventa, con el impulso de la oferta a través de programas europeos de apoyo a la diversificación de la economía rural que alentaron el crecimiento de la actividad.

La Palma ha sido una isla pionera en la implantación de la oferta de turismo rural en el conjunto de Canarias, teniendo en la Asociación de Turismo Rural Isla Bonita, creada en 1992, una de las primeras entidades de su naturaleza constituida en el archipiélago y en el conjunto de España, para el impulso y desarrollo de la actividad. A su vez, el turismo rural en la isla de La Palma se encuentra inserto en la estrategia de desarrollo rural de la iniciativa comunitaria LEADER (Relaciones entre Actividades de Desarrollo de la Economía Rural), que gestiona, como Grupo de Acción Local, la Asociación para el Desarrollo Rural de la isla de La Palma (ADER-La Palma) desde el año 1991.

Por su parte, en España, el turismo rural comenzó a cobrar protagonismo a través del Programa nacional de vacaciones en "casas de labranza" en 1968 y, posteriormente, en los años ochenta, con la concesión de subvenciones públicas estatales para el acondicionamiento de viviendas de actores locales que se convirtieron en incipientes promotores del turismo rural (Bote, 1992). El paulatino aumento de los alojamientos turísticos en el medio rural se vio impulsado por el programa de financiación europea de la iniciativa LEADER en 1991 y sus diversas ediciones posteriores (Fernández Hernández, 2008). Esta iniciativa fomentaba la revitalización y diversificación de las economías rurales de forma innovadora y participativa, teniendo como una de sus líneas de acción el apoyo a actividades de fomento del turismo rural a través de inversiones económicas orientadas a una amplia gama de acciones.

El trabajo realizado en esta investigación se centra en el estudio de la oferta de alojamiento de turismo rural en la isla de La Palma. Partiendo del registro oficial de establecimientos de turismo rural disponible en la Consejería de Turismo y Transportes del Cabildo Insular de La Palma, se identificó un total de 195 casas rurales con 234 unidades de alojamiento y 829 plazas turísticas. Estas casas rurales se encuentran registradas por 161 titulares de su explotación y están distribuidas en la totalidad de los municipios de la isla de La Palma.

De este universo de establecimientos, se pudo contactar con el 92,8\% de las casas rurales, es decir, 181 establecimientos turísticos, y el 93,5\% de todas las unidades de alojamiento. De esta forma, se entrevistó al $95,6 \%$ del total de aquellas personas registradas como propietarias o, en su caso, las personas encargadas de la gestión de las casas rurales, lo cual se traduce en 154 gestores y gestoras de los establecimientos turísticos. Los encuentros personales se basaron en la administración de un cuestionario acerca de la actividad del turismo rural en la isla por parte de una persona encuestadora, cuya duración fue de en torno a quince minutos. El trabajo de campo se llevó a cabo entre el 15 de febrero y el 14 de mayo del año 2007.

Para el desarrollo del trabajo empírico de este estudio, se recurrió a técnicas de investigación cuantitativa como método de recogida de información primaria. El cuestionario ha sido 
la herramienta utilizada. Las preguntas realizadas fueron compiladas a través de una combinación de respuestas abiertas y de selección múltiple, las cuales versan en torno a las siguientes variables:

- características de la persona promotora: género, ocupación principal, dedicación exclusiva o no a la actividad, sector de compatibilización de la actividad.

- nivel de dedicación laboral requerida: trabajo directo, personas implicadas en el desempeño de la actividad, horas dedicadas a la gestión de la oferta de turismo rural por parte de la persona promotora y otro personal implicado, ingresos obtenidos a través de la actividad.

Para llevar a cabo el análisis de los datos recabados en los cuestionarios administrados, se ha utilizado la herramienta estadística SPSS. De manera transversal, el análisis de los datos se ha realizado bajo la impronta de la perspectiva de género, para lo cual se ha trabajado con las distintas variables distinguiendo la tendencia general de la tendencia particular en función del género.

En cuanto a los análisis específicos de las variables abordadas, se ha llevado a cabo contrastes de hipótesis, así como análisis del grado de asociación entre variables, utilizando el coeficiente $\mathrm{V}$ de Cramer, la prueba de Levene, y la prueba de T para comparación de la igualdad de medias, estableciendo o no diferencias significativas entre variables. Para la interpretación de las inferencias, se ha determinado el nivel de significación del análisis en $5 \%(\alpha=0,05)$.

\section{RESULTADOS Y DISCUSIÓN}

Para el análisis empírico del acceso a la autonomía económica de las mujeres a través del ejercicio del turismo rural en la isla de La Palma, se profundiza comparativamente en el perfil profesional de mujeres y hombres promotores, a la par que se estudia su dedicación laboral para el desarrollo de la actividad, con el fin de poder encontrar diferencias significativas entre ambos géneros.

\subsection{Perfil profesional de la persona promotora de turismo rural en la isla de La Palma}

La caracterización de las personas promotoras de turismo rural se realiza teniendo en cuenta, principalmente, su situación profesional en relación con la gestión de la actividad turística que realizan. Las variables abordadas se analizan en función del género de las personas promotoras de turismo rural, permitiendo reconocer la existencia o no de diferencias de género en torno a la actividad en la isla de La Palma.

En primer lugar, se observa que del total de las 154 personas estudiadas, 87 promotores son hombres frente a las 67 mujeres promotoras de la actividad de turismo rural. Esto se traduce en un $56,5 \%$ de hombres y un $43,5 \%$ de mujeres. Además de tener en cuenta la propensión a la mayor masculinización del medio rural, los datos obtenidos evidencian la realidad de que los hombres en la isla de La Palma ostentan la titularidad de los alojamientos turísticos y gestionan la actividad económica del turismo rural en mayor medida que las mujeres, lo cual resulta coherente con la menor tradición empresarial que registran las mujeres en 
los distintos sectores económicos (Censo de Población y Vivienda, 2011), así como con las mayores dificultades de acceso a recursos económicos y patrimoniales que tradicionalmente han obstaculizado su asunción de un emprendimiento propio.

A pesar de ello, el relevante $43,5 \%$ de mujeres promotoras de turismo rural refleja una importante proporción de mujeres que han encontrado en la actividad turística de la isla una oportunidad laboral, lo que es coherente con la literatura revisada que muestra el hecho de que el autoempleo femenino en turismo rural ha sido una estrategia para enfrentar las dificultades que tienen las mujeres en su acceso a empleos remunerados en los entornos rurales (Alonso et al., 2014; Caballé Rivera, 2000; García-Ramón et al., 1995; Kiper et al., 2011; Pallarès et al., 2015).

\subsubsection{Ocupación principal de las personas promotoras de turismo rural}

Las personas promotoras de turismo rural de la isla de La Palma desarrollan diversos tipos de ocupaciones que definen la situación profesional en que se encuentran. Del total de personas encuestadas, solo un $15,6 \%$ se define como empresariado en turismo rural. Destaca que un $19,5 \%$ son personas agricultoras o ganaderas de explotaciones de pequeña dimensión, valor que sitúa esta ocupación como la más frecuente entre quienes promueven el turismo rural. De otro lado, un $13,6 \%$ se define bajo la categoría de funcionariado, mientras que un $12,3 \%$ son personas empresarias o autónomas con cinco o menos trabajadores/as a su cargo. Asimismo, un $11 \%$ es personal obrero cualificado. Paralelamente, se observa un $10,4 \%$ de personas en situación de jubilación y con dedicación a la gestión de alojamientos de turismo rural. Las personas promotoras dedicadas a "labores domésticas" representan tan solo un 1,9\%, mientras que aquellas que se encuentran desempleadas configuran un 1,3\% del total de las personas gestoras de turismo rural.

El análisis de la ocupación principal en función del género aporta algunas particularidades. De esta forma, se encuentra que mientras un $27,6 \%$ del total de hombres promotores son agricultores o ganaderos de pequeñas explotaciones, solo un $9 \%$ del total de mujeres promotoras tiene a las actividades agropecuarias como su principal ocupación. Por otra parte, un $14,9 \%$ de los hombres son autónomos o empresarios con cinco o menos trabajadores a su cargo, mientras que de las mujeres promotoras solo el $9 \%$ se desempeña en esta categoría profesional. A su vez, mientras que un $17,9 \%$ de las mujeres son funcionarias, un $10,3 \%$ de los hombres promotores se encuentran en esa misma situación profesional. Además, un relevante $26,9 \%$ de las mujeres promotoras se definen como empresarias de turismo rural, frente al 6,9\% de los hombres promotores que se manifiestan bajo esta situación profesional.

Asimismo, se observa que el 1,3\% de personas que se declaran en situación de desempleo corresponden exclusivamente a hombres. A su vez, el 1,9\% de personas declaradas bajo la categoría de "labores domésticas" son mujeres. En ambos contextos se elude la consideración de empresarios o empresarias en la actividad turística, prevaleciendo la situación como desempleados o como amas de casa.

Al estudiar la existencia de una relación significativa entre la ocupación principal y el género de los promotores, se observa que el estadístico $V$ de Cramer toma el valor $V=0,455$, 
que, al estar acotado entre 0 y 1 , indica la existencia de asociación entre dichas variables cualitativas. Además, para confirmar lo anterior, se lleva a cabo un contraste de hipótesis con el objetivo de comprobar si la asociación que existe es significativa o no. Se obtiene un p-valor o significación $p<0,001$ menor que el nivel de significación fijado, por lo que se rechaza la hipótesis nula de independencia entre las variables, pudiendo afirmar que el género de la persona promotora influye significativamente en la ocupación principal en la que se emplea.

De aquí que en esta investigación se confirme la división de género del mercado de trabajo para el grupo de personas promotoras de turismo rural estudiado en la isla de La Palma, observándose una marcada segregación de género según el tipo de actividad que desarrollan. En el cuadro 1 se pueden observar las diferentes proporciones de hombres y mujeres registradas en las distintas ocupaciones, de manera que los varones destacan sobre las mujeres como empleados de actividades agropecuarias de pequeñas explotaciones, mayoritariamente, y como autónomos, obreros cualificados, profesionales liberales y desempleados. Por el contrario, la presencia de las mujeres es más concurrida, en comparación con los hombres, en la categoría de empresarias de turismo rural, principalmente, y como funcionarias y empleadas en "labores domésticas" o amas de casa.

Cuadro 1. Ocupación principal según el género de la persona promotora de turismo rural

\begin{tabular}{|l|c|c|}
\hline \multirow{2}{*}{} & \multicolumn{2}{|c|}{ Sexo } \\
\cline { 2 - 3 } & Hombre & Mujer \\
\hline No sabe/No contesta & & $3,0 \%$ \\
\hline Labores domésticas & & $4,5 \%$ \\
\hline Desempleado/a & $2,3 \%$ & \\
\hline Jubilado/a & $9,2 \%$ & $11,9 \%$ \\
\hline Agricultor/a, ganadero/a pequeña explotación & $27,6 \%$ & $9,0 \%$ \\
\hline Autónomo/a o empresario/a (5 o $<$ trab.) & $14,9 \%$ & $9,0 \%$ \\
\hline Autónomo/a o empresario/a (6 o > trab.) & $3,4 \%$ & $1,5 \%$ \\
\hline Profesional liberal & $4,6 \%$ & $3,0 \%$ \\
\hline Otros empleados de oficina & $3,4 \%$ & $4,5 \%$ \\
\hline Directivo/a (6 o > subordinados) & $4,6 \%$ & \\
\hline Obreros cualificados & $12,6 \%$ & $9,0 \%$ \\
\hline Funcionario/a & $10,3 \%$ & $17,9 \%$ \\
\hline Empresario/a de turismo rural & $6,9 \%$ & $26,9 \%$ \\
\hline Total & $100 \%$ & $100 \%$ \\
\hline Estadístico V de Cramer: $V=0,455 ; p=0,001$ & & \\
\hline
\end{tabular}

Estos datos son coherentes con otros estudios abordados en la literatura revisada, en los cuales se alude a la segregación de género del mercado laboral, especialmente, acusada en los entornos rurales (Alario et al., 2008; Alonso et al., 2014; Millán Vázquez et al., 2017). Por una parte, se observa la masculinización de los trabajos agropecuarios, hecho que está relacionado con el tradicional papel de "ayuda familiar" ejercido por las mujeres en este sector productivo, desempeñando las tareas requeridas sin el reconocimiento económico, social y 
de derechos correspondiente (Vera Toscano, 2009). Por otro lado, se observa la significativa mayor presencia de mujeres que de hombres cuya principal ocupación es el turismo rural, lo cual se mantiene en línea con la fundamental participación de las mujeres en esta actividad mencionada por diferentes autores (Brandth et al., 2011; Caballé Rivera, 2000; Dávila et al., 2011; García-Ramón et al., 1995), debido al tipo de labores domésticas y de atención al visitante que requiere.

\subsubsection{Exclusividad de la dedicación a la gestión de la casa rural}

En cuanto a si la persona promotora se dedica exclusivamente a la gestión de la actividad de turismo rural o, por el contrario, compatibiliza con otras ocupaciones, se evidencia que un $86,3 \%$ no se dedica de forma exclusiva a la gestión de las casas rurales, frente al $13,7 \%$ que sí lo hace. Esta amplia brecha entre los porcentajes obtenidos es indicativo de la dificultad de las personas promotoras para acceder a la autonomía económica a través del turismo rural, actividad que implica se haya de recurrir a otras ocupaciones para garantizar una fuente completa de ingresos, debido al carácter discontinuo de la demanda que limita la obtención de ingresos estables, por su naturaleza de pequeña escala y dimensión económica. Considerado de otro modo, el turismo rural en el contexto insular estudiado, para personas promotoras que gestionan una única casa rural, solo puede ser considerado como una fuente de obtención de una renta complementaria.

Cuando se realiza este análisis atendiendo a la diferencia de género de las personas promotoras, se encuentra que un $90,5 \%$ de las personas que respondieron que sí se dedicaban exclusivamente a la actividad de turismo rural son mujeres, frente al 9,5\% de hombres. Por otra parte, en el grupo de promotores y promotoras que respondieron que no se dedicaban exclusivamente al turismo rural, se encuentra un $64,4 \%$ hombres por un $35,6 \%$ mujeres. Los datos expresan cómo las mujeres son más propensas que los hombres a tener como única actividad económica la gestión de las casas rurales. Por el contrario, son más los hombres que las mujeres quienes tienen otras actividades económicas más allá de la oferta de actividades de turismo rural.

Al recurrir al estadístico $V$ de Cramer, se obtiene el valor $V=0,381$, lo que indica la existencia de asociación. Este hecho se confirma con el $p$-valor o significación $p<0,001$, lo cual lleva a rechazar la hipótesis nula de independencia entre las variables estudiadas. Por tanto, la dedicación exclusiva o no al turismo rural depende, de manera significativa, del género de la persona que desempeña la actividad.

Las características propias del turismo rural, marcadas por la estacionalidad y la discontinuidad de la demanda turística, impide la obtención de una renta completa a través de la actividad, los resultados en cuanto a la mayor propensión de las mujeres promotoras a dedicarse de forma exclusiva al turismo rural están en línea con las referencias de la literatura que sitúan a las mujeres en mayor medida que los hombres en trabajos temporales y a tiempo parcial, con su consiguiente sobrerrepresentación en condiciones de precariedad laboral y baja remuneración (Millán Vázquez et al., 2017, Moreno Mínguez, 2003; Vera Toscano, 2009), lo cual obstaculiza su capacidad de acceso a una verdadera autonomía económica. 


\subsubsection{Actividad según sector compatibilizado con el turismo rural}

En lo que respecta al sector de actividad económica con la que las personas promotoras compatibilizan la actividad de turismo rural, un $48,5 \%$ ejercen en el sector servicios, mientras que un $25 \%$ lo hace en el sector agropecuario. Bajo la categoría de "otras situaciones" se declara un $15,2 \%$, mientras que un $7,6 \%$ y un $3,8 \%$ de las personas promotoras se emplean en el sector de la construcción y de la industria, respectivamente.

Al estudiar el sector de actividad con el que se compatibiliza la gestión de turismo rural según el género, destaca que mientras el $29,4 \%$ de los hombres promotores compagina la gestión de la casa rural con labores agropecuarias, solo lo hace el $17 \%$ de las mujeres. Asimismo, las mujeres promotoras participan en un $59,6 \%$ en el sector servicios, por el $42,4 \%$ de hombres. Estos datos confirman la mayor tendencia de las mujeres a ocuparse principalmente en el sector servicios, mientras que los hombres, a pesar de ejercer su actividad económica mayoritariamente también en el sector servicios, se encuentran representados en mayor proporción que las mujeres en el resto de sectores económicos. Esta realidad fue referida en la literatura revisada, en la cual se menciona la segregación horizontal del mercado de trabajo remunerado, y la tendencia a la feminización del sector servicios, donde las mujeres se ocupan en trabajos relacionados con el servicio y el cuidado (Alario et al., 2008; Cànoves Valiente et al., 2008; Millán Vázquez et al., 2017), labores relacionadas con el trabajo doméstico que tradicionalmente han ejercido en sus hogares (Rijkers et al., 2012).

Una mayor presencia de hombres entre el conjunto de las personas promotoras de turismo rural en La Palma se traduce en ocupar en mayor medida que las mujeres los distintos sectores de actividad económica. Sin embargo, el estadístico $V$ de Cramer, nos proporciona un valor bajo, $V=0,244$. Al realizar el contraste, se obtiene un $p$-valor $=0,096$, que no llega a establecer una relación significativa entre el sector con el que se compatibiliza la actividad de turismo rural y el género de la persona promotora, confirmando la hipótesis de independencia entre las variables.

\subsection{Trabajo y dedicación al turismo rural en la Isla de la Palma}

En este apartado se analiza el trabajo que realizan las personas promotoras para la gestión de la casa rural, así como la implicación de otras personas en este cometido. Además, se estudia el tiempo dedicado por los promotores y promotoras, y otras personas vinculadas a la actividad, en las diversas tareas que la gestión del establecimiento de turismo rural requiere en la isla de La Palma.

\subsubsection{Trabajo directo de la persona promotora en la casa rural}

En primer lugar, interesa determinar si las personas promotoras trabajan directamente en la gestión de la casa rural o si, por el contrario, delegan las tareas en terceras personas bajo cualquier fórmula de prestación de servicios. Mayoritariamente, son las personas promotoras directamente quienes se encargan de la atención que requiere la actividad alojativa rural. Así, el $89,5 \%$ de los promotores y promotoras trabaja directamente en la gestión de la casa rural, frente a un $10,5 \%$ que no administra directamente la casa rural. 
Al abordar esta variable desde la perspectiva de género, se ha de notar que el $90,6 \%$ de los hombres y el $88,1 \%$ de las mujeres promotoras se implican directamente en la gestión de la casa rural, lo cual refleja proporciones similares entre ambos géneros. En efecto, el estadístico de Cramer, con un valor $V=0,041$ y una significación o $p$-valor $=0,614$, no establece diferencias significativas, por lo que la variable del género no influye en que las personas promotoras de turismo rural trabajen o no directamente en la casa rural.

\subsubsection{Personas con desempeño en la gestión de la casa rural}

Las tareas de gestión de una casa rural pueden recaer en una o varias personas, por lo que se pretende conocer cuántas personas están vinculadas a esta actividad. Los datos reflejan que en el 50,6\% de las casas rurales hay dos personas con implicación en el desempeño de la actividad, seguido de un $22,4 \%$ de casas en las que se implican para su gestión tres personas. Un $21,8 \%$ de las casas tiene a una persona como única responsable y prestataria de servicios. A pesar del carácter no intensivo de los servicios que requiere un alojamiento rural y de la flexibilidad que puede entrañar su gestión, a la luz de los datos obtenidos se observa que de modo efectivo requiere de más de una persona para el desempeño de la actividad. Puesto que se trata de una actividad con arraigado componente familiar, parece lógico suponer que estas personas vinculadas podrían ser integrantes de la misma familia de la persona promotora.

Desagregando bajo la perspectiva de género el análisis realizado, el cuadro 2 muestra que en un $29,4 \%$ de las casas rurales con promotores varones se implican dos personas, frente al $21,2 \%$ de los emprendimientos que son promovidos por mujeres. A su vez, en un $14,7 \%$ de las casas rurales promovidas por varones se implican en su gestión a tres personas, frente al $7,7 \%$ de las casas rurales promovidas por mujeres. Por el contrario, cuando sólo hay una persona encargada de atender la casa rural, un $8,2 \%$ de estas casas rurales tienen promotores varones, por un $13,5 \%$ de casas cuyas promotoras son mujeres.

Estos datos parecen reflejar la mayor propensión de los emprendimientos de turismo rural regentados por hombres a contar con más de una persona para la atención de las casas rurales. No obstante, el estadístico $\mathrm{V}$ de Cramer, con un valor de $\mathrm{V}=0,219$ y una significación o $p$-valor $=0,148$, no alcanza a establecer diferencias significativas entre el género y el número de personas implicadas en la atención de la casa rural.

Cuadro 2. Personas implicadas en la atención de la casa rural según el género de la persona promotora

\begin{tabular}{|l|l|c|c|c|c|c|c|}
\hline \multirow{2}{*}{} & \multicolumn{7}{|c|}{ Personas implicadas en la atención de la casa rural } \\
\cline { 3 - 8 } \multicolumn{2}{|c|}{} & $\mathbf{1}$ & $\mathbf{2}$ & $\mathbf{3}$ & $\mathbf{4}$ & $\mathbf{5}$ & $\mathbf{7}$ \\
\hline \multirow{2}{*}{ Sexo } & Hombre & $8,2 \%$ & $29,4 \%$ & $14,7 \%$ & $2,4 \%$ & $0,6 \%$ & $0,6 \%$ \\
\cline { 2 - 8 } & Mujer & $13,5 \%$ & $21,2 \%$ & $7,6 \%$ & $1,8 \%$ & & \\
\hline \multicolumn{2}{|l}{ Estadístico V de Cramer: V = 0,219; $\mathrm{p}=0,148}$. \\
\hline
\end{tabular}

La mayor tendencia de los alojamientos turísticos gestionados por varones a contar con más de una persona implicada en la atención de la casa rural puede relacionarse con el tipo 
de labores domésticas que requiere la actividad, tal y como se refirió en la literatura (GarcíaRamón et al., 1995; Rodríguez Muñoz y Acevedo Duarte, 2015). De este modo, las tareas a desempeñar pueden ser percibidas como propias del género femenino, al ser similares a las tareas que las mujeres han asumido tradicionalmente en los hogares, $y$, por ende, que las mismas sean delegadas en otras mujeres externas o de la propia unidad familiar.

\subsubsection{Tiempo de dedicación a la gestión de la casa rural}

A la hora de analizar el tiempo que se emplea en la gestión del turismo rural, y dado que en gran medida es una tarea discontinua y flexible, se ha considerado el cómputo de horas dedicadas a las diversas tareas de gestión de la actividad por semana por cada unidad de alojamiento. Se observa que las personas promotoras de las casas rurales dedican una media de 6,3 horas a la semana a gestión de la actividad, mientras que el resto de personal implicado lo hace en 10,9 horas por semana. Se ha de destacar, en primer lugar, la exigencia de dedicación a la semana que requiere la gestión de una unidad de alojamiento de turismo rural (17,2 horas), que se aproxima en su conjunto a media jornada laboral. A su vez, se observa un mayor nivel de dedicación asignado al personal implicado en la atención de la casa rural en comparación con la de los promotores y promotoras, lo cual parece indicar que el personal colaborador en las tareas de gestión asume una parte sustancial del volumen de trabajo que el alojamiento genera.

En el análisis de género, las mujeres dedican al turismo rural una media de 5,7 horas frente a las 6,8 horas semanales dedicadas por los hombres. Con respecto al personal implicado en la gestión de la casa rural, en los emprendimientos turísticos promovidos por hombres se observa que el personal implicado dedica una media de 11,8 horas, mientras que en las casas rurales cuyas promotoras son mujeres, el personal implicado dedica 9,7 horas de trabajo. En conjunto, se observa una mayor cantidad de tiempo dedicado en las casas rurales promovidas por hombres, tanto en la dedicación que ellos asumen en su gestión, como en la dedicación que tiene lugar cuando otras personas tienen participación en la actividad. Esto podría estar relacionado con la naturaleza doméstica de las tareas requeridas en la gestión de las casas rurales, estando las mujeres tradicionalmente más familiarizadas en función de los roles de género asumidos y desempeñados en el ámbito familiar, lo que llevaría a una mayor eficiencia en el uso del tiempo. No obstante, se requeriría de un estudio más detallado de las actividades desempeñadas en la gestión del alojamiento rural para obtener resultados más concluyentes.

A pesar de ello, la prueba de Levene sobre la igualdad de las varianzas en las horas semanales dedicadas a la gestión de la casa rural de hombres y mujeres promotoras, con un valor $F=3,855$ y una significación o $p$-valor $p=0,051$, no alcanza a establecer diferencias en función del género al asumir que las varianzas son iguales. Asimismo, la prueba T para la igualdad de medias, para el caso de varianzas iguales, mantiene una significación de p-valor = 0,315 , lo cual confirma la igualdad de las medias del número de horas dedicadas a la semana por hombres y mujeres promotoras.

A su vez, la prueba de Levene sobre las horas semanales dedicadas por el personal implicado a la gestión de las casas rurales de hombres y mujeres promotores expresa un valor $F=2,332$ y una significación o $p$-valor $=0,129$, por lo que se asume que las varianzas son 
iguales. Además, la prueba de T para la igualdad de medias, para el caso de varianzas iguales, tiene un $p$-valor $=0,146$, de forma que se confirma la hipótesis de igualdad de las horas medias dedicadas por otro personal implicado en casas rurales promovidas tanto por hombres como por mujeres.

\subsubsection{Ingresos obtenidos a través del turismo rural}

Bajo la perspectiva de que la actividad de alojamiento en el turismo rural representa una renta complementaria para sus promotores y promotoras, interesa conocer cuál es la contribución a su renta personal. Los datos obtenidos del estudio empírico, ante la pregunta a las personas promotoras del porcentaje de ingresos que tal actividad representa en el total de ingresos personales, arroja una estimación del $28,3 \%$ de los ingresos procedentes de la gestión de los alojamientos de turismo rural en la isla de La Palma.

Cuando se analiza el porcentaje de ingresos en función del género de los promotores, se encuentra que las mujeres tienden a recibir un mayor volumen de ingresos personales a través del turismo rural que los hombres. En este sentido, las mujeres obtienen un $37,1 \%$ del total de sus ingresos a partir del trabajo realizado en la actividad turística, frente al $21,2 \%$ del total de los ingresos de los hombres promotores de turismo rural. Las diferencias entre los ingresos recibidos del turismo rural por mujeres y hombres promotores resultan ser significativas. De esta forma, se puede afirmar que el turismo rural contribuye a una mayor proporción de los ingresos personales de las mujeres promotoras con respecto a la proporción de ingresos que el turismo rural aporta a la renta de los hombres promotores, cuestión que se relaciona con la referencia aportada en la literatura sobre las mayores dificultades encontradas por las mujeres en el mercado de trabajo remunerado del medio rural (Censo de Población y Vivienda, 2011; Millán Vázquez et al., 2017, Moreno Mínguez, 2003; Vera Toscano, 2009). Esta realidad dificulta su acceso a actividades que les reporten mayores ingresos económicos que la actividad del turismo rural, que, como se ha visto, no alcanza a generar una renta completa, frente al caso de los varones, que participan en mayor medida de otras actividades que les permiten obtener mayores beneficios económicos.

Al tomar en cuenta las horas de trabajo dedicadas por otro personal implicado en la gestión de la casa rural diferente de las personas promotoras, se observan algunas particularidades en función del género. De esta manera, en el caso de las casas rurales cuyos promotores son hombres, la correlación lineal es $r=0,579$, con un $p$-valor $<0,001$, lo cual establece una correlación significativa entre el porcentaje de ingresos y el número de horas trabajadas por otro personal implicado. Esto se traduce en que a mayor número de horas trabajadas por el personal implicado en las casas rurales gestionadas por hombres, mayor es el porcentaje de ingresos que estos reciben por la actividad. Sin embargo, en lo que respecta a las casas rurales promovidas por mujeres, no se produce esta relación entre las variables, en tanto la correlación de Pearson tiene un $p$-valor $p=0,333$. Por consiguiente, el porcentaje de ingresos obtenidos por la gestión del turismo rural por parte de las mujeres promotoras no se relaciona con el número de horas de trabajo que realizan otras personas implicadas en la casa rural.

Estos datos ofrecen una perspectiva de la mayor dependencia de los hombres con respecto al trabajo realizado por otras personas implicadas en la actividad turística, cuyo trabajo 
contribuye a aumentar la rentabilidad económica de la actividad de turismo rural que gestionan. A su vez, sugiere que la contribución del trabajo de otras personas en los alojamientos regentados por mujeres tiene un impacto menor en su rendimiento, lo cual podría ser un indicativo de diferencias de productividad, de cuyo origen sería preciso ahondar.

\section{CONCLUSIONES}

A partir del marco teórico estudiado, y en contraste con los resultados de la investigación empírica, se pueden establecer algunas conclusiones pertinentes. En primer lugar, se constata que el número de mujeres emprendedoras de turismo rural en la isla de La Palma es inferior al número de hombres promotores, lo cual es coherente con la menor tradición empresarial de las mujeres y las menores oportunidades laborales y de emprendimiento registradas en los entornos rurales. Esto se relaciona con los distintos usos del tiempo y el espacio que se realizan por razón de género. En efecto, las mujeres, tradicionalmente, han estado confinadas al espacio privado, como las principales proveedoras de los cuidados familiares y las labores domésticas, lo cual ha dificultado su acceso a recursos económicos y patrimoniales, así como su éxito profesional y la asunción de cargos de responsabilidad laboral.

En cuanto al perfil profesional de las personas promotoras, se observa una significativa mayor proporción de mujeres que de hombres cuya ocupación principal es el turismo rural. Es decir, dedicarse de forma exclusiva al turismo rural encuentra diferencias significativas en función del género de la persona que impulsa la actividad, de manera que son las mujeres las que se dedican en mayor medida al turismo como su principal actividad económica. Por consiguiente, los hombres promotores participan en mayor proporción de otras actividades productivas que les reportan otros ingresos económicos. Además, entre los promotores y promotoras de turismo rural se observa una marcada segregación de su participación en el mercado de trabajo remunerado, situándose en profesiones distintas en función de su género.

Por otro lado, considerando el desempeño realizado para la gestión de la actividad turística, se observa que las personas promotoras mayoritariamente participan de forma directa en las actividades necesarias en el alojamiento rural. Sin embargo, más de la mitad de los casos estudiados cuentan con dos o más personas implicadas en la atención de la casa rural. En este sentido, del trabajo resulta que son los hombres promotores quienes delegan un mayor número de horas de trabajo en otras personas implicadas en la actividad. No obstante, en el caso de los hombres promotores que recurren a otras personas para la realización de las tareas de mantenimiento de la casa rural, no excluye la posibilidad de que sean mujeres las principales encargadas de las labores de turismo rural. Este aspecto relativo al estudio del conjunto de personas implicadas en la actividad, bajo una perspectiva de género, requiere de un análisis posterior, debido a las limitaciones de este estudio en cuanto a la identificación del perfil del conjunto de personas involucradas en la actividad, más allá del promotor o promotora.

En lo que respecta al nivel de ingresos, los datos estudiados confirman que el turismo rural es una actividad que no reporta altos ingresos económicos, lo cual se vincula con la relativa baja ocupación de este tipo de turismo. En esta línea, se ha observado que existen diferencias significativas en cuanto a los ingresos aportados por el turismo rural entre las 
mujeres y los hombres promotores. El porcentaje de ingresos recibidos está en estrecha relación con el hecho de que el turismo sea o no la principal ocupación de sus promotores y promotoras, lo cual afecta en mayor medida a las mujeres que a los hombres. De esta manera, son las mujeres las que manifiestan recibir un mayor porcentaje de sus ingresos a partir del turismo rural, lo cual se relaciona con que los hombres participan, en mayor medida, de otras actividades productivas que les reportan otras entradas económicas. No obstante, pese a que el turismo rural aporta a las mujeres unos ingresos económicos, que para un grupo de ellas se conforma como la única fuente de ingresos disponibles, no son lo suficientemente amplios como para garantizar una renta completa que permita su independencia económica a través de la actividad, de manera que, aunque el turismo rural ha permitido a las mujeres acceder a una fuente de ingresos, esta actividad productiva no es suficiente como para garantizar su autonomía económica.

A fin de revertir la situación de desigualdad de género acusada especialmente en el mercado laboral remunerado de los entornos rurales, la oportunidad de un turismo rural fortalecido y acrecentado por una progresiva profesionalización de la actividad, así como nutrido con ofertas de servicios añadidos o complementarios distintos de la estancia, habría de repercutir en la obtención de unos rendimientos económicos superiores que pudieran aproximarse a una renta completa. Estos ingresos económicos deben crecer en paralelo al incremento de valor recibido por los servicios en el conjunto de la experiencia de los turistas. Ofertas más elaboradas, propuestas de alojamientos bajo tematización e interpretación basados en distintos motivos y argumentos, estrategias de venta cruzada con productos locales, incorporación de los establecimientos de alojamiento en circuitos de productos e itinerarios locales, entre otros. La revalorización con ofertas que acrecienten los servicios básicos han de ser una condición para que las mujeres promotoras mejoren su situación laboral y, por consiguiente, su autonomía económica, a través del turismo rural.

Entre las contribuciones de este trabajo, los resultados sostienen como certeza la segregación de género existente en el mercado laboral, con el cariz específicamente estudiado para el medio rural. Las evidencias obtenidas afianzan el hecho de una presencia significativa mayor de mujeres que de hombres ejerciendo el turismo rural como principal ocupación, aspecto ampliamente reflejado en la literatura. La segregación horizontal, concentrándose la ocupación de las mujeres en mayor medida que los hombres en el sector servicios, se pone de manifiesto, alineándose esta posición con actividades de servicios y cuidados desempeñados tradicionalmente en el ámbito del hogar. Si bien, en lo sustancial, el turismo rural no altera el modelo de desempeño imperante, nutriéndose de unas experiencias adquiridas por las mujeres en el ámbito doméstico, supone un avance en el grado de su autonomía y autorrealización. Ambos aspectos han de ser considerados desde una política pública que aliente la participación femenina en la actividad, contribuyendo al cambio de mentalidad desde el modelo patriarcal, favoreciendo estrategias que permitan la participación efectiva en la actividad y fomentando procesos de formación, recualificación y rediseño de experiencias turísticas rurales que permitan involucrar todo el potencial de desarrollo que la mujer aporta en el medio rural. 


\section{REFERENCIAS BIBLIOGRÁFICAS}

Alario, M., Baraja, E., y Pascual, H. (2008). Género y espacio: la inserción laboral de las mujeres rurales, en Maya Frades, V. (Ed.). Mujeres Rurales. Estudios multidisciplinares de género. Ediciones Universidad de Salamanca, Salamanca, 61-80.

Alonso, N. \& Trillo, D. (2014). Women, rural environment and entrepreneurship. Social and Behavioral Sciences, 161, 149-155. doi:https://doi.org/10.1016/j.sbspro.2014.12.039

Annes, A., \& Wright, W. (2015). Creating a room of one's own: French farm women, agritourism and the pursuit of empowerment. Women's Studies International Forum, 53, 1-11. doi:https://doi.org/10.1016/j.wsif.2015.08.002

Anthopoulou, T. (2010). Rural women in local agrofood production: Between entrepreneurial iniciatives and family strategies. A case study in Greece. Journal of Rural studies, 26, 394-403. doi:https://doi.org/10.1016/j.jrurstud.2010.03.004

Bote Gómez, V. (1992). Turismo en Espacio Rural. Rehabilitación del patrimonio sociocultural y de la economía local. Madrid: Editorial Popular, S.A.

Brandth, B., \& Haugen, M. S. (2010). Doing Farm Tourism: The Intertwining Practices of Gender and Work. Journal of Women in Culture and Society, 35 (2), 426-446. doi:https://doi. org/10.1086/605480

Brandth, B., Haugen, M. S., \& Kroken, A. (2011). Farm tourism: a question of gender and competence? Journal of Agricultural Science and Technology, B No.1, 1-10. Recuperado de https://www.researchgate.net/publication/253241101 Farm Tourism A Question of Gender and Competence

Caballé Rivera, A. (2000). Implicaciones de género en el desarrollo de la oferta de agroturismo en Navarra y Asturias. En García Ramón, M.D. y Baylina Ferré, M. (eds.), El nuevo papel de las mujeres en el desarrollo rural. Oikos-tau, Barcelona, 153-169.

Cànoves Valiente, G., y Blanco Romero, A. (2008). El papel de las mujeres en la diversificación de actividades económicas en una comarca rural de Cataluña: El caso del Pallars Jussà. Revista SEMATA, 20, 133-154. http://hdl.handle.net/10347/4524

Dávila, A. M, Pinuer, M., y Szmulewicz, P. (2011). Análisis comparativo de la situación laboral de las mujeres rurales: Vitivinicultura, Industria Vitivinícola y Turismo Enológico. El caso del Valle de Colchagua, Chile. Rosa dos Ventos, 3 (2), 266-275. Recuperado de http:// www.ucs.br/etc/revistas/index.php/rosadosventos/article/view/1252

Diéguez, M. I., Gueimonde, A., y Sinde, A.I. (2010). Éxito empresarial y género en turismo rural. Tourism \& Management Studies, 6, 82-93. Recuperado de Dialnet: https://dialnet.unirioja.es/servlet/articulo?codigo $=3887980$

Feng, X. (2013). Women's Work, Men's Work: Gender and Tourism among the Miao in Rural China. Anthropology of Work Review, XXXIV (1), 2-14. doi:http://doi.org/10.1111/ awr.12002

Fernández Hernández, C. (2008). El turismo rural como factor de dinamización del medio rural. En Fernández Hernández, C. y Martín Torres, M.F. (coords.). Planificación y gestión del turismo rural. Reflexiones desde la experiencia en Canarias (pp. 37-76). Santa Cruz de Tenerife: Federación Canaria de Desarrollo Rural.

Forstner, K. (2013). Women's Group-based Work and Rural Gender Relations in the Southern Peruvian Andes. Bulletin of Latin American Research, 32 (1), 46-60. doi:https://doi. org/10.1111/i.1470-9856.2011.00693.x 
García-Ramón, M. D., Cànoves, G., Salamaña, I., Valdovinos, N., y Villamarino, M. (1995). Trabajo de la mujer, turismo rural y percepción del entorno: una comparación entre Cataluña y Galicia. Agricultura y Sociedad, 75, 115-152. Recuperado de Dialnet: https:// dialnet.unirioja.es/servlet/articulo?codigo $=82986$

García Sanz, B. (2009). Ocupación y rentas de las mujeres en el medio rural. Agricultura familiar en España 2009, 142-152. Recuperado de https://www.upa.es/anuario_2009/ pag 142-152 benjamingarcia.pdf

Gómez García, J.M., y Rico González, M. (2005). La mujer en el medio rural de Castilla y León: Diversificación sectorial y proceso de dinamización económica. Revista de Estudios de Economía Aplicada, 23 (2), 465-490. Recuperado de Dialnet: https://dialnet.unirioja. es/descarga/articulo/1250457.pdf

lakovidou, O. (2002). Women's Agrotourist Cooperatives in Greece: Key Elements for Their Successful Operation. Journal of Rural Cooperation, 30 (1), 13-24. Recuperado de http://ageconsearch.tind.io/record/60881

Khatiwada, L. K., \& Silva, J. A. (2015). Mitigating Gender Inequality in Rural Regions: The Effects of Tourism Employment in Namibia. International Journal of Tourism Research, 17, 442 450. doi:https://doi.org/10.1002/itr.2010

Kiper, T., Ozdemir, G., \& Basaran, B. (2011). Applicability of agricultural tourism and the role of women. A case study of Sarkoy-Murefte. Journal of Environmental Protection and Ecology, 12 (3), 1146-1159. Recuperado de https://docs.google.com/a/jepe-journal. info/viewer?a=v\&pid=sites\&srcid=amVwZS1ab3VybmFsLmluZm98amVwZS1ab3VybmFsfGd4OjJkMDdmOTQ3Y2FINTA3MzI

Langreo Navarro, A., y Benito García, I. (2005). La mujer en la agricultura y en el medio rural. Agricultura Familiar en España, 104-128. Recuperado de http://www.fademur.es/ documentos/pag 104-128 langreo.pdf

Little, J., \& Panelli, R. (2003). Gender Research in Rural Geography. Gender, Place and culture, 10 (3), 281-289. doi:http://dx.doi.org/10.1080/0966369032000114046

Millán Vázquez de la Torre, M.A.G., Velasco Portero, M.T., y Ramírez Sobrino, J.N. (2017). EI emprendimiento de la mujer rural española: análisis de la brecha salarial. Una realidad difícil de solucionar. Papeles de Población, 23 (92), 151-183. doi:http://dx.doi.org/10.2 2185/24487147.2017.92.016

Moreno Mínguez, A. (2003). La situación laboral de la mujer rural en Castilla y León y Extremadura: un análisis sociológico. Revista Acciones e Investigaciones Sociales, 17,109153. Recuperado de Dialnet: https://dialnet.unirioja.es/descarga/articulo/698108.pdf

Pallarès, M., Tulla, A. \& Vera, A. (2015). Environmental capital and women's entrepreneurship: a sustainable local development approach. Carpathian Journal of Earth and Environmental Sciences, 10 (3), pp.133-146. Recuperado de https://www.researchgate. net/publication/280255149 Environmental capital and women\%27s entrepreneurship A sustainable local development approach

Rijkers, B \& Costa, R. (2012). Gender and Rural Non-Farm Entrepreneurship. World Development, 40 (12), 2411-2426. http://dx.doi.org/10.1016/i.worlddev.2012.05.017

Rodríguez Muñoz, G., y Acevedo Duarte, A. (2015). Cambios en la vida cotidiana de las mujeres a través de la incorporación al trabajo turístico en el Rosario, Michoacán, México. 
El Periplo Sustentable: Revista de turismo, desarrollo y competitividad, 29, 5-33. Recuperado de Dialnet: $\underline{\text { https://dialnet.unirioja.es/servlet/articulo?codigo=5165305 }}$

Sampedro, R., y Camarero, L. (2007). Mujeres empresarias en la España rural: El sujeto pendiente del desarrollo. Revista Internacional de Sociología (RIS), LXV (48), 121-146. Recuperado de http://revintsociologia.revistas.csic.es/index.php/revintsociologia/ article/view/71/72

Suárez Ortega, M. (2016). Across gender. Work situations of rural women in the South of Spain. Qualitative Research in Education, 5 (1), 77-104. doi:http://dx.doi.org/10.17583/ gre.2016.1814

Tajeddini, K., Ratten, V., \& Denisa, M. (2017). Female tourism entrepreneurs in Bali. Journal of Hospitality and Tourism Management, 31, 52-58. doi:https://doi.org/10.1016/i. ihtm.2016.10.004

Vera Toscano, E. (2009). Empleo femenino y bienestar económico en el medio rural. Agricultura familiar en España 2009, 134-141. Recuperado de https://www.upa.es/anuario 2009/ pag 134-141 esperanzavera.pdf

Viruela Martínez, R., y Domingo Pérez, C. (2000). Mujer y trabajo en el contexto regional español. En García Ramón, M.D. y Baylina Ferré, M. (eds.), El nuevo papel de las mujeres en el desarrollo rural, 65-90. Barcelona: Oikos-tau.

Wright, W., \& Annes, A. (2014). Farm Women and Agritourism: Representing a New Rurality. Sociologia Ruralis, 54, 477-499. doi:https://doi.org/10.1111/soru.12051

Wilkinson, P. F., \& Pratiwi, W. (1995). Gender and Tourism in an Indonesian Village. Annals of Tourism Research, 22 (2), 283-299. doi:https://doi.org/10.1016/0160-7383(94)00077-8 